Research Article

\title{
Polarization Properties of Obliquely Incident EM Waves in Nonuniform Weakly Ionized Dusty Plasma
}

\author{
Li-Ping Gan $\mathbb{D}^{\infty}$, Li-Xin Guo $\mathbb{D}^{\infty}$, Jiang-Ting Li $\mathbb{D}^{\infty}$, and Lin-Jing Guo \\ School of Physics and Optoelectronic Engineering, Xidian University, Xi'an 710071, China \\ Correspondence should be addressed to Li-Xin Guo; lxguo@xidian.edu.cn
}

Received 26 May 2021; Accepted 14 September 2021; Published 5 October 2021

Academic Editor: Muhammad Zubair

Copyright (c) $2021 \mathrm{Li}$-Ping Gan et al. This is an open access article distributed under the Creative Commons Attribution License, which permits unrestricted use, distribution, and reproduction in any medium, provided the original work is properly cited.

\begin{abstract}
Effects of nonuniform weakly ionized dusty plasma on the polarization properties of obliquely incident electromagnetic (EM) waves were theoretically investigated in this paper. The dielectric coefficient of magnetized dusty plasma is obtained based on the Bhatnagar-Gross-Krook collision model. Then, the effects of external magnetic field, dust radii, and density on the polarization mismatch loss of obliquely incident EM waves in the $L$-band were analyzed using the equivalent impedance method. Results indicate that the larger the external magnetic field, the greater the polarization mismatch loss. In addition, we also found that dusty particles alleviate the deterioration of the polarization state significantly; regulating and controlling dust radius and density can avoid polarization reversal and reduce the polarization mismatch loss. We further analyzed the variation of the polarization state under different reentry heights. Our analysis reveals interesting features concerning polarization properties of obliquely incident EM waves, which provide an important theoretical basis for information reception in the plasma sheath containing ablative particles.
\end{abstract}

\section{Introduction}

When a hypersonic vehicle flies near space, a complex nonuniform dusty plasma will form on the surface of the vehicle because of high temperature, which will cause radar signal attenuation and lead to communication blackout $[1,2]$. Compared with ordinary plasma, the properties of dusty plasma are more complex due to the existence of dust particles. The collisions of dust particles with electrons or ions lead to complex interactions between dusty plasma and EM waves [3]. The problem of EM waves propagate in nonuniform dusty plasma has been concerned by many scholars. Karasev et al. [4] investigated the features of dusty plasma in a strongly inhomogeneous magnetic field. Liu et al. [5] discussed the attenuation characteristics of $\mathrm{THz}$ circularly polarized wave in nonuniform dusty plasma. They found that the attenuation of the right-hand circular polarized (RHCP) wave and the left-hand circular polarized (LHCP) wave in magnetized dusty plasma is different. Jia et al. [6] researched the attenuation of EM waves in fully ionized dusty plasma. Chen et al. [7, 8] adopted the FDTD method which analyzed the attenuation and scattering properties of EM waves in nonuniform and time-varying dusty plasma.

Nevertheless, these studies have a number of limitations. For one thing, most of these research studies regarded the collision frequency as uniform, while the collision frequency in plasma is nonuniform, especially the plasma sheath around a hypersonic vehicle [9-11]. For another, only fewer research studies considered the variation of the polarization state of obliquely incident EM waves in the plasma medium. In the nonuniform dusty plasma medium, waves with different polarization states experience different attenuations in amplitude and phase. Thus, there is a change in transmitted waves, which may induce a mismatch loss between the receiving antenna and the transmitted signals.

The polarization properties of obliquely incident EM waves propagating in nonuniform plasma can now be investigated. Yin et al. [12] analyzed the transmission and polarization properties of EM waves in inhomogeneous plasma slabs. They pointed out that nonuniform magnetized plasma slabs would lead to the transmitted wave 
experiencing two kinds of waves. Shahrokhi et al. [13] researched the polarization evolution of EM waves in motional plasma and proposed a novel diagnostic method. However, the polarization properties in nonuniform weakly ionized dusty plasma have not been considered. Hence, discussing the polarization properties of EM waves in nonuniform weakly ionized and magnetized dusty plasma is of great importance.

In this paper, the equivalent impedance method was used to calculate the axial ratio, the phase difference, and the polarization mismatch loss under different external magnetic fields and different dusty plasma parameters in the $L$ band. Particularly, the distributions of electron density and collision frequency are inhomogeneous. Moreover, the polarization state of EM waves for eight reentry heights was analyzed. The rest of this paper is organized as follows. Section 2 presents the theoretical basis. Then, in Section 3, the effects under different parameters on the polarization characteristics of EM waves in nonuniform weakly ionized dusty plasma are investigated. Finally, in Section 4, the conclusions are obtained.

\section{The Theoretical Basis}

2.1. Magnetized and Weakly Ionized Dusty Plasma Model. Here, the nonuniform weakly ionized dusty plasma we analyzed is regarded as "cold" plasma. Charged particles in magnetized dusty plasma will be subjected to the Lorentz force, which will result in a cyclotron motion [14]. The electron cyclotron frequency is

$$
\omega_{c e}=\frac{q B}{m_{e}} .
$$

In (1), $m_{e}$ and $q$ denote the electron mass and electron charge, and $B$ represents the magnetic field intensity. The kinetic equation of magnetized dusty plasma [15] is

$$
\frac{\partial f^{(\alpha)}}{\partial t}+v \cdot f^{(\alpha)}+\frac{q}{m_{\alpha}}\left(E+\frac{1}{c} v \times B\right) \cdot \nabla_{v} f^{(\alpha)}=\left(\frac{\partial f^{(\alpha)}}{\partial t}\right)_{\mathrm{col}},
$$

where $f^{(\alpha)}$ represents the distribution function of charged particles, $v$ denotes the velocity of charged particles, $E$ is the electric field intensity, and $c$ is the speed of light in vacuum. According to the BGK collision model, the Boltzmann equation can be expressed as

$$
\frac{\partial f^{(e)}}{\partial t}+\frac{q}{m_{e}}\left(E+\frac{1}{c} v \times B\right) \frac{\partial f^{(e)}}{\partial v}=\left(\frac{\partial f^{(e)}}{\partial t}\right)_{B-G-K},
$$

where $f^{(e)}$ is the electron distribution function and $\left(\partial f^{(e)} / \partial t\right)_{B-G-K}$ is the BGK collision term [16]. The electron distribution function can be decomposed into the equilibrium component and perturbation component, $f^{(e)}(\nu)=$ $f_{0}(v)+f_{1}(v)$ where

$$
f_{0}=n_{e}\left(\frac{m_{e}}{2 \pi \kappa T_{e}}\right)^{3 / 2} \exp \left(\frac{-m_{e} \nu^{2}}{2 \pi \kappa T_{e}}\right)
$$

is the equilibrium component. $n_{e}$ is the electron density; $\kappa$ is the Boltzmann constant; $T_{e}$ denotes the electronic temperature. The perturbation component under the magnetization condition can be obtained by solving the Boltzmann equation [17]:

$$
f_{1}(v, t)=\frac{j q n_{e} E}{2 m_{e}(\pi)^{3 / 2}\left(\omega+\omega_{c e}-j v_{e n}(v)\right)}\left(\frac{m_{e}}{\kappa T_{e}}\right)^{2} t e^{-t^{2}} .
$$

In (5), $v_{e n}$ is the collision frequency; $\omega$ represents the incident wave frequency; $t=v \sqrt{m_{e} / 2 \kappa T_{e}}$. The incident current caused by the collision between electrons and ions can be expressed as $j_{e}=q \int_{0}^{\infty} v f_{1} \mathrm{~d} v$. Furthermore, based on Ohm's law, the complex conductivity under the external magnetic field is

$$
\sigma=\varepsilon_{0} \frac{\omega_{p}^{2} v_{e n}}{\left(\left(\omega+\omega_{c e}\right)^{2}+v_{e n}^{2}\right)}-j \omega \varepsilon_{0} \frac{\omega_{p}^{2}}{\left(\left(\omega+\omega_{c e}\right)^{2}+v_{e n}^{2}\right)},
$$

where $\omega_{p}=\sqrt{n_{e} q^{2} / m_{e} \varepsilon_{0}}$ and $\varepsilon_{0}$ represents the permittivity in vacuum. Then, the effects of dusty particles are taken into consideration. Combining the finite orbit [18], the current resulting from the collision of dust particles and electrons is

$$
I_{e}=-q \int_{0}^{\infty} v \sigma_{q}^{d} f_{1}(v) \mathrm{d} v
$$

where $\sigma_{q}^{d}=\pi r_{d}^{2}, r_{d}$ is the dust radius.

Finally, according to the current continuity law [19-21] and equations (6) and (7), the dielectric constant of magnetized dusty plasma in weak ionization and weak collision is

$$
\begin{aligned}
\varepsilon(\omega)= & 1-\frac{\omega_{p}^{2}\left(\omega+\omega_{c e}\right)}{\omega\left[\left(\omega+\omega_{c e}\right)^{2}+v_{e n}^{2}\right]}+\frac{c \eta_{e d}\left(\omega+\omega_{c e}\right)}{\varepsilon_{0} \omega\left[\left(\omega+\omega_{c e}\right)^{2}+v_{e n}^{2}\right]\left[\left(\omega+\omega_{c e}\right)^{2} v_{c h}^{2}\right]} \\
& -\frac{j}{\omega}\left\{\frac{\omega_{p}^{2} v_{e n}}{\left[\left(\omega+\omega_{c e}\right)^{2}+v_{e n}^{2}\right]}+\frac{c \eta_{e d}\left(\omega+\omega_{c e}\right)-v_{e n} v_{c h}}{\varepsilon_{0}\left[\left(\omega+\omega_{c e}\right)^{2}+v_{e n}^{2}\right]\left[\left(\omega+\omega_{c e}\right)^{2} v_{c h}^{2}\right]}\right\},
\end{aligned}
$$


where $\eta_{e d}=\pi q^{2} r_{d}^{2} n_{e} n_{d} / m_{e}$ is the charging response factor, $n_{d}$ represents the density of dust particles, and $v_{c h}$ is the charging frequency.

The plasma electron density in real space can be seen as bi-Gaussian distribution [22]. The collision frequency and the electron temperature have a linear relationship [23, 24]. So, we consider that the collision frequency and the plasma temperature experience the same distribution. Then, the electron density and collision frequency are defined as follows:

$$
\begin{aligned}
n_{e}(z) & = \begin{cases}n_{0} \exp \left[-a_{1}\left(z-z_{0}\right)^{2}\right], & \left(0 \leq z<z_{0}\right), \\
n_{0} \exp \left[-a_{2}\left(z-z_{0}\right)^{2}\right], & \left(z \geq z_{0}\right),\end{cases} \\
v_{e n}(z) & =v_{0} \exp \left(-b\left(z-z_{1}\right)^{2}\right),
\end{aligned}
$$

where $n_{0}$ and $v_{0}$ denote the peak value of electron density and collision frequency. $a_{1}, a_{2}$ and $b$ are the rising and decreasing coefficients of electron density and collision frequency. $z_{1}$ and $z_{0}$ represent the position where $n_{e}$ and $v_{e n}$ get the maximum values. For the convenience of calculation, the values of $a_{1}$ and $a_{2}$ are $0.05 / \mathrm{cm} ; z_{0}$ and $z_{1}$ take the middle position of the plasma model; the value of $b$ is 5 .

It is so difficult to obtain an accurate analytical solution when EM waves propagate in inhomogeneous dusty plasma. However, inhomogeneous dusty plasma can be

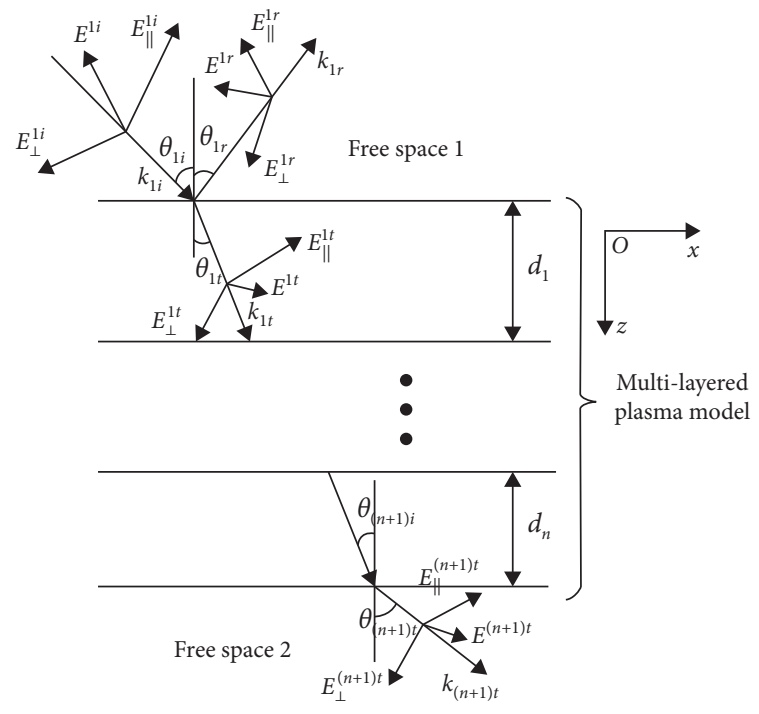

FIGURE 1: EM waves propagating in stratified plasma.

approximated by some homogeneous stratified structures. Here, the inhomogeneous dusty plasma is divided into $n$ layers. In each layer, dusty plasma can be seen as uniform. Thus, the permittivity of the magnetized and weakly ionized dusty plasma at layer $m$ is

$$
\begin{aligned}
\varepsilon_{m}(\omega)= & 1-\frac{\omega_{p m}^{2}\left(\omega+\omega_{c e}\right)}{\omega\left[\left(\omega+\omega_{c e}\right)^{2}+v_{e n m}^{2}\right]}+\frac{c \eta_{e d m}\left(\omega+\omega_{c e}\right)}{\varepsilon_{0} \omega\left[\left(\omega+\omega_{c e}\right)^{2}+v_{e n m}^{2}\right]\left[\left(\omega+\omega_{c e}\right)^{2} v_{c h}^{2}\right]} \\
& -\frac{j}{\omega}\left\{\frac{\omega_{p m}^{2} v_{\text {enm }}}{\left[\left(\omega+\omega_{c e}\right)^{2}+v_{e n}^{2}\right]}+\frac{c \eta_{e d m}\left(\omega+\omega_{c e}\right)-v_{e n} v_{c h}}{\varepsilon_{0}\left[\left(\omega+\omega_{c e}\right)^{2}+v_{e n m}^{2}\right]\left[\left(\omega+\omega_{c e}\right)^{2} v_{c h}^{2}\right]}\right\},
\end{aligned}
$$

where $\omega_{p m}, v_{e n m}, v_{c h m}$, and $\eta_{e d m}$ denote the plasma frequency, the collision frequency, the charging frequency, and the charging response factor in layer $m$, respectively.

2.2. Polarization Theory. When EM waves are obliquely incident on the plasma medium, they can be decomposed into a parallel polarized wave and perpendicular polarized wave, as shown in Figure 1 . Here, $E^{m i}, E^{m t}$, and $E^{m r}$ represent the incident wave, transmitted wave, and reflected wave, respectively, $m=1,2, \ldots, n+1 ; k_{m i}, k_{m t}$, and $k_{m r}$ are propagation constants of them, $k_{m i}=k_{m r} ; \theta_{m i}, \theta_{m t}$, and $\theta_{m r}$ are the incident angle, transmitted angle, and reflected angle, respectively, $\theta_{m i}=\theta_{m r} ; d_{m}$ is the thickness of layer $m$. The transmitted wave can be expressed as a combination of the parallel polarization wave and perpendicular polarization wave.

$$
\begin{aligned}
E^{(n+1) t} & =E^{(n+1) t} \mathbf{u}+E_{\perp}^{(n+1) t} \mathbf{u}_{\perp} \\
& =E^{1 i} T \mathbf{u}+E_{\perp}^{1 i} T_{\perp} \mathbf{u}_{\perp} .
\end{aligned}
$$

In (11), $E_{\|}^{(n+1) t}$ and $E_{\perp}^{(n+1) t}$ are components of the parallel polarized wave and vertical polarized wave, respectively. $T_{\|}$and $T_{\perp}$ are transmission coefficients of the parallel polarized wave and vertical polarized wave. The equivalent impedance method can be used to calculate the transmission coefficients of the parallel polarized wave and perpendicular polarized wave [25]. $T_{\|}$and $T_{\perp}$ are given in Appendix. $\mathbf{u}_{\|}$and $\mathbf{u}_{\perp}$ denote unit vectors of the parallel polarized wave and vertical polarized wave.

EM waves with different polarization states experience different attenuations in amplitude and phase. So, the polarization state of EM waves will change after passing through nonuniform dusty plasma. According to the polarization theory of EM waves [26], the polarization characteristics of EM waves can be well expressed by axial ratio. Its expression is

$$
\mathrm{AR}=\tan \left(\frac{1}{2} \arcsin \left(\frac{2\left|E^{(n+1) t}\right|\left|E_{\perp}^{(n+1) t}\right| \sin \phi^{t}}{\left|E^{(n+1) t}\right|^{2}+\left|E_{\perp}^{(n+1) t}\right|^{2}}\right)\right) .
$$


In (12), $\phi_{t}$ is the phase difference between the parallel polarized wave and the perpendicular polarized wave. Its expression is

$$
\begin{aligned}
\phi^{t} & =\phi_{\perp}^{t}-\phi^{t} \\
& =\arg \left(E_{\perp}^{(n+1) t}\right)-\arg \left(E^{(n+1) t}\right) \pm{ }^{90^{\circ}} .
\end{aligned}
$$

In (13), $\phi_{\perp}^{t}$ and $\phi_{\|}^{t}$ are the phases of the vertical polarized wave and the parallel polarized wave, respectively. \pm represents that the EM wave is LHCP or RHCP. The calculation results of axial ratio can be used to indicate the polarization state of the transmitted wave. If the value is 0 , the transmitted wave is circular polarization. When the value is infinity, the transmitted wave is linear polarization. Other values represent elliptical polarization. In addition, we stipulate that a positive (negative) axial ratio value denotes a left-handed (right-handed) polarization.

The variation of the polarization state may cause a polarization mismatch loss between the transmitted wave and the receiving antenna. The Stokes parameters can describe polarization characteristics of the EM wave more comprehensively [26]. The expressions of Stokes parameters are

$$
\left\{\begin{array}{l}
g_{0}^{t}=\left|E^{(n+1) t}\right|^{2}+\left|E_{\perp}^{(n+1) t}\right|^{2} \\
g_{1}^{t}=\left|E^{(n+1) t}\right|^{2}-\left|E_{\perp}^{(n+1) t}\right|^{2} \\
g_{2}^{t}=2 \cdot\left|E^{(n+1) t}\right| \cdot\left|E_{\perp}^{(n+1) t}\right| \cos \phi^{t} \\
g_{3}^{t}=2 \cdot\left|E^{(n+1) t}\right| \cdot\left|E_{\perp}^{(n+1) t}\right| \sin \phi^{t}
\end{array}\right.
$$

We presume that the Stokes parameters of the receiving antenna are

$$
\left[\begin{array}{llll}
g_{0}^{r} & g_{1}^{r} & g_{2}^{r} & g_{3}^{r}
\end{array}\right]
$$

Then, using (14) and (15), the polarization match coefficient is

$$
m_{p}=\frac{g_{0}^{r} g_{0}^{t}+g_{1}^{r} g_{1}^{t}+g_{2}^{r} g_{2}^{t}+g_{3}^{r} g_{3}^{t}}{2 \cdot g_{0}^{r} g_{0}^{t}}
$$

The polarization match coefficient describes the degree of the polarization matching between the receiving antenna and transmitted signals. Furthermore, the mismatch loss between the receiving antenna and the transmitted signals is

$$
\text { loss }=10 \cdot \log _{10}\left(m_{p}\right) \mathrm{dB} \text {. }
$$

Therefore, the polarization state and the mismatch loss can be analyzed using (12), (13), and (17).

\section{Results and Discussion}

Our focus is to analyze polarization properties of obliquely incident EM waves through the nonuniform weakly ionized dusty plasma. So, the differences in the axial ratio, the phase difference, and the polarization mismatch loss under different parameters were discussed. The incident wave and the receiving antenna are both $\mathrm{RHCP}$. For all of the following numerical examples, the incident wave frequency is $2 \mathrm{GHz}$. In this section, dust radius and dust density are homogeneous. All numerical parameters of Figures 2-4 are shown in Table 1 . These parameters are given according to presented references [6, 27].

3.1. Algorithmic Verification. In order to prove the correctness of our calculation, we compared results of the axial ratio with presented reference [28]. Here, $n_{0}=1 \times 10^{18} / \mathrm{m}^{3}$, and the collision frequency is $1 \mathrm{GHz}$. The numerical result is shown in Figure 5. The black line is the calculation result using the equivalent impedance method, and the red dots are the calculated results of the transmission line method. Figure 5 shows that the calculation results of the two methods are consistent. So, the method used in this paper is feasible.

3.2. Effects of Different Magnetic Field Intensities. Here, we discuss the polarization properties of EM waves under different magnetic field intensities. The axial ratio, the phase difference, and the polarization mismatch loss under different magnetic field intensities are presented in Figure 2.

For $B=0 \mathrm{~T}$, Figure 2(a) shows that the axial ratio first decreases from 0 to a negative value and then changes from negative to positive when incident angle is $84^{\circ}$. These changes indicate that the polarization state of EM waves first changes from RHCP to the right-hand elliptically polarized wave and then transforms into the left-hand elliptically polarized wave. In other words, the polarization reversal appears when incident angle is $84^{\circ}$. For $B=0.5 \mathrm{~T}$, the polarization reversal appears when incident angle is $61^{\circ}$. For $B=1 \mathrm{~T}$, the polarization reversal appears when incident angle is $54^{\circ}$. That is, the external magnetic field aggravates the depolarization of EM waves. The presented reference [12] also pointed out that the external magnetic field can change the polarization state of EM waves, and the larger the cyclotron frequency is, the smaller the transmission of copolarization is. In addition, Figure 2(b) shows that the values of the phase difference of perpendicular polarized waves and parallel polarized waves contain positive and negative. This further demonstrates that the transmitted waves undergo two types of polarization state.

What is most noticeable in Figure 2(c) is that the variation of the polarization state causes a certain polarization mismatch loss under different magnetic fields. The larger the magnetic field intensity, the greater the polarization mismatch loss. In other words, the larger the magnetic field intensity, the more the transmitted waves cannot be received because of polarization mismatch loss.

3.3. Effects of Different Dusty Plasma Parameters. Dust radius and density have obvious effects on the propagation of EM waves in nonuniform dusty plasma. Therefore, the axial ratio, the phase difference, and the polarization mismatch loss under different dusty parameters are calculated. The numerical results are presented in Figures 3 and 4.

Figure 3 indicates that dust particles significantly alleviate the deterioration of the polarization state of EM 


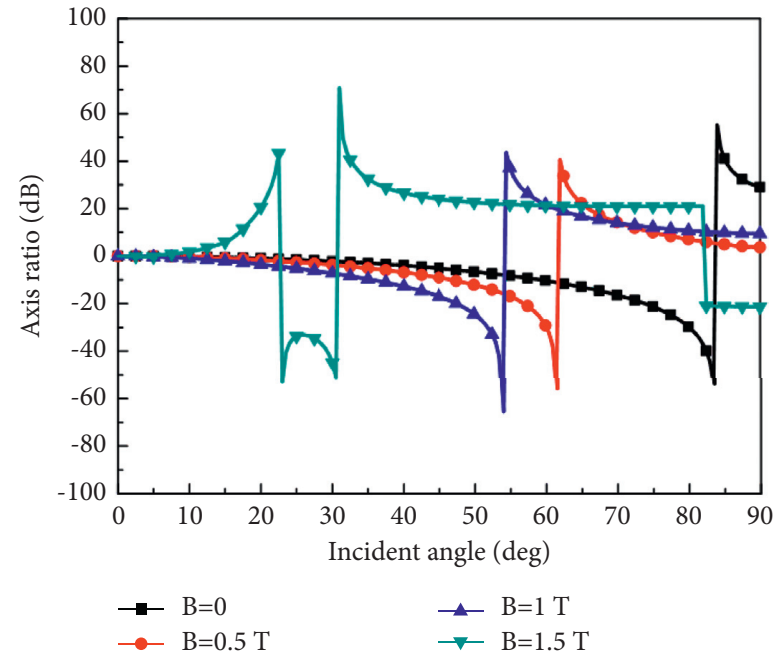

(a)

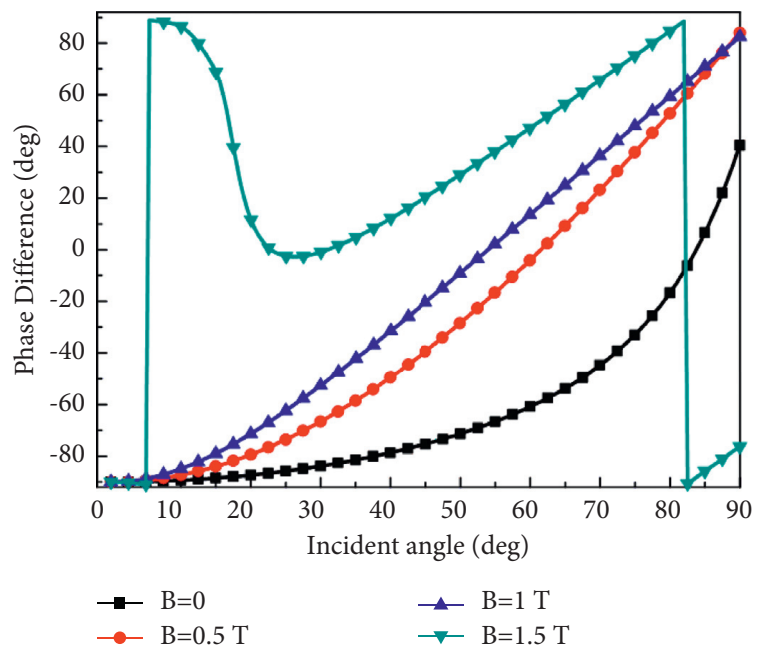

(b)

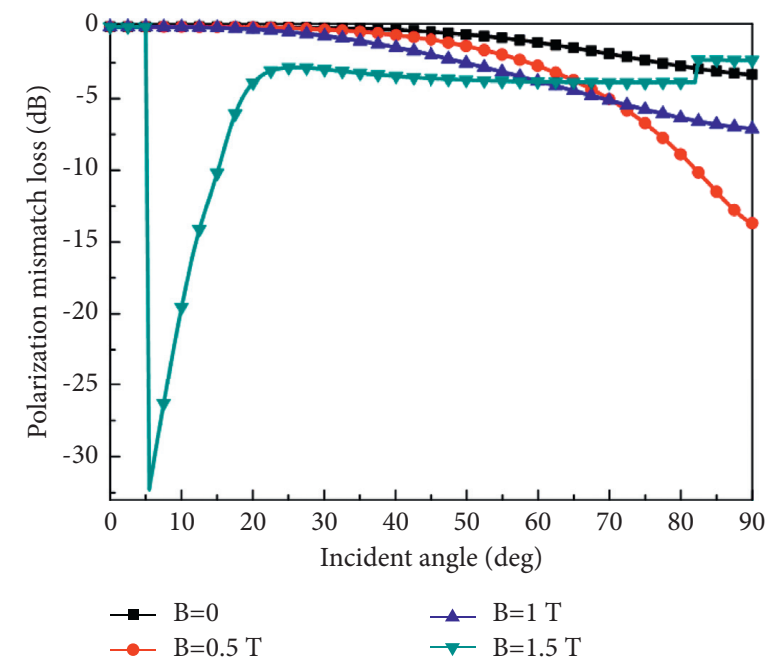

(c)

Figure 2: Effects of different magnetic field intensities versus the incident angle: (a) axial ratio, (b) phase difference, and (c) polarization mismatch loss.

TABLe 1: Calculation parameters in Figures 2-4.

\begin{tabular}{|c|c|c|c|c|c|c|}
\hline & $n_{0}\left(\mathrm{~m}^{-3}\right)$ & $v_{0}(\mathrm{GHz})$ & $r_{d}(\mu \mathrm{m})$ & $n_{d}\left(\mathrm{~m}^{-3}\right)$ & $v_{c h}(\mathrm{GHz})$ & $B(\mathrm{~T})$ \\
\hline Figure 2 & $1 \times 10^{18}$ & 1 & 3 & $3 \times 10^{12}$ & 0.22 & $0.0 .5,1,1.5$ \\
\hline Figure 3 & $1 \times 10^{18}$ & 1 & $0,1,3,5$ & $3 \times 10^{12}$ & 0.22 & 0 \\
\hline Figure 4 & $1 \times 10^{18}$ & 1 & 3 & $0,1 \times 10^{12}, 3 \times 10^{12}, 5 \times 10^{12}$ & 0.22 & 0 \\
\hline
\end{tabular}

waves. For plasma without dust, the transmitted wave becomes a left-hand elliptically polarized wave when incident angle is $81^{\circ}$. When considering the effects of dusty particles, Figures $3(\mathrm{a})$ and $3(\mathrm{~b})$ indicate that polarization reversal appears only in case where the dust radius is $1 \mu \mathrm{m}$. There is no polarization reversal that occurs when the dust radius is $3 \mu \mathrm{m}$ and $5 \mu \mathrm{m}$. In addition, Figure 3(c) also shows that dust particles cause reduction in the polarization mismatch loss.

What is the most important is that the variation of the polarization mismatch loss increased when the dust radius increases from $3 \mu \mathrm{m}$ to $5 \mu \mathrm{m}$. So, for dusty plasma, there is an optimal dust radius that minimizes the polarization mismatch loss.

Figure 4 indicates that dust density has the same effects as the dust radius. Dust particles can alleviate the depolarization effect of EM waves and reduce the polarization mismatch loss. Similarly, a suitable dust density can avoid the phenomenon of polarization reversal. Therefore, reasonable regulation and controlling of the radius and density of dust particles in plasma can effectively reduce the loss caused by the polarization 


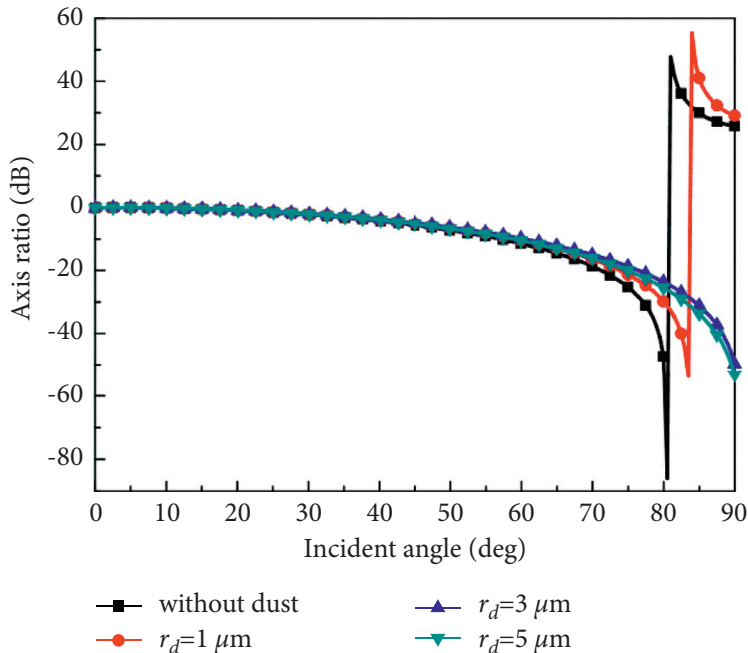

(a)

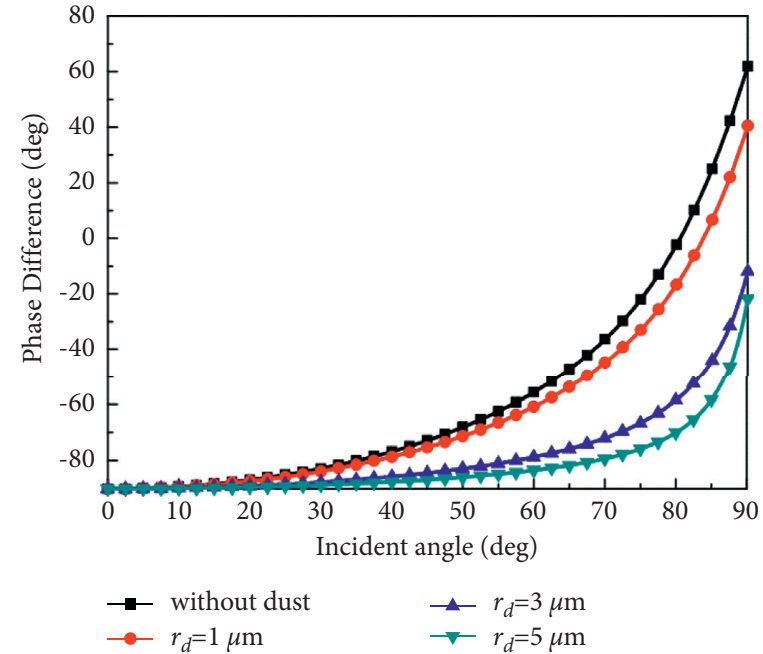

(b)

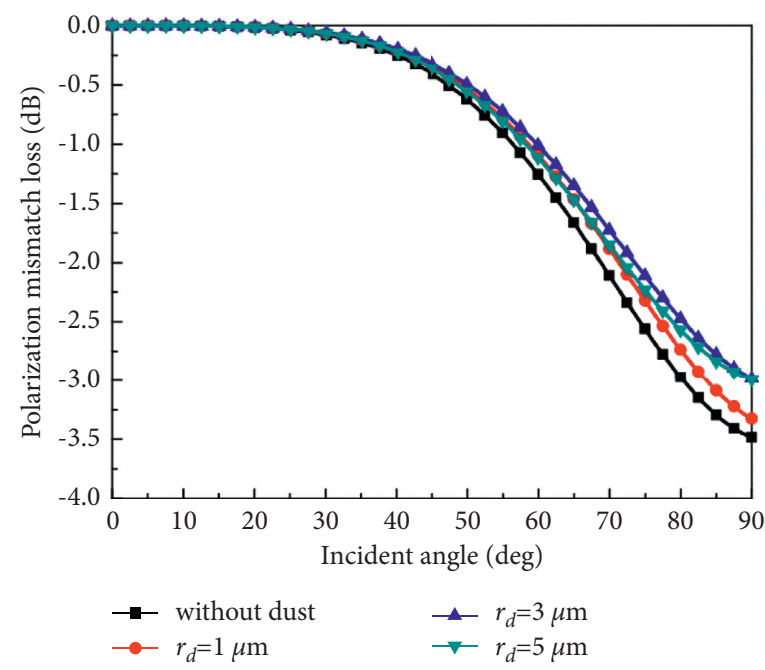

(c)

Figure 3: Effects of different values of dust radius on EM waves versus the incident angle: (a) axial ratio, (b) phase difference, and (c) polarization mismatch loss.

mismatch between the transmitted signals and the receiving antenna.

3.4. Effects of Different Reentry Heights. The electron density and distributions and the peak values of collision frequency of eight reentry heights in the NASA experiment [29] are adopted to investigate the polarization properties of EM waves caused by nonuniform weakly dusty plasma. Other parameters are $r_{d}=3 \mu \mathrm{m}, \quad n_{d}=3 \times 10^{12} / \mathrm{m}^{3}$, and $v_{c h}=0.22 \mathrm{GHz}$. Results are presented in Figure 6 .
Clearly, Figure 6 confirms that there is a certain mismatch loss at each reentry height. From Figure 6(a), we can see that the polarization state of transmitted waves changes at each reentry height. The variation of the polarization state is the maximum at $30 \mathrm{~km}$ and the minimum at $70 \mathrm{~km}$. Then, Figure 6(b) shows that the phase difference at each height ranges from $-90^{\circ}$ to $0^{\circ}$, which indicates that the transmitted waves at each height are always in the right-handed polarized wave. Finally, the polarization mismatch loss shows the order $30 \mathrm{~km}>25 \mathrm{~km}>47 \mathrm{~km}>53 \mathrm{~km}>61 \mathrm{~km}>21 \mathrm{~km}>71 \mathrm{~km}>76$ $\mathrm{km}$, which is consistent with the axial ratio in Figure 6(a). In 


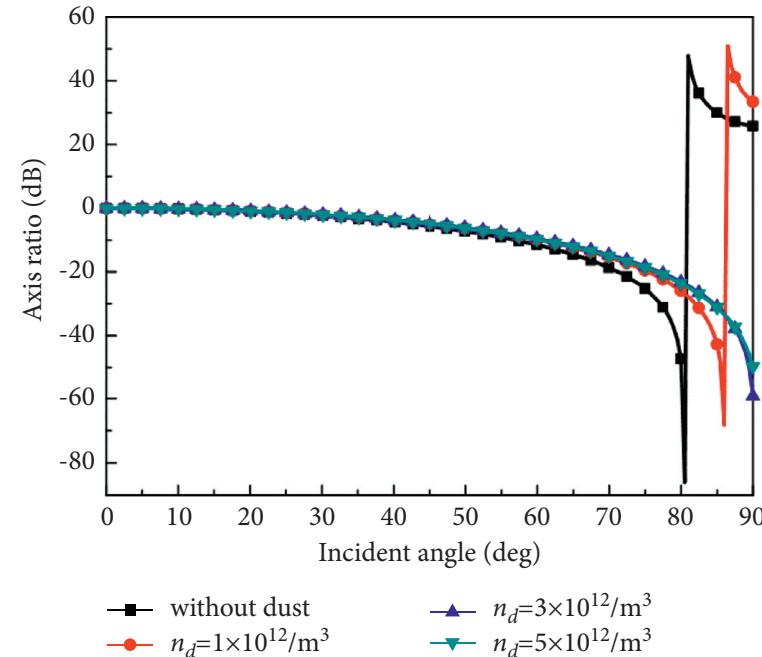

(a)

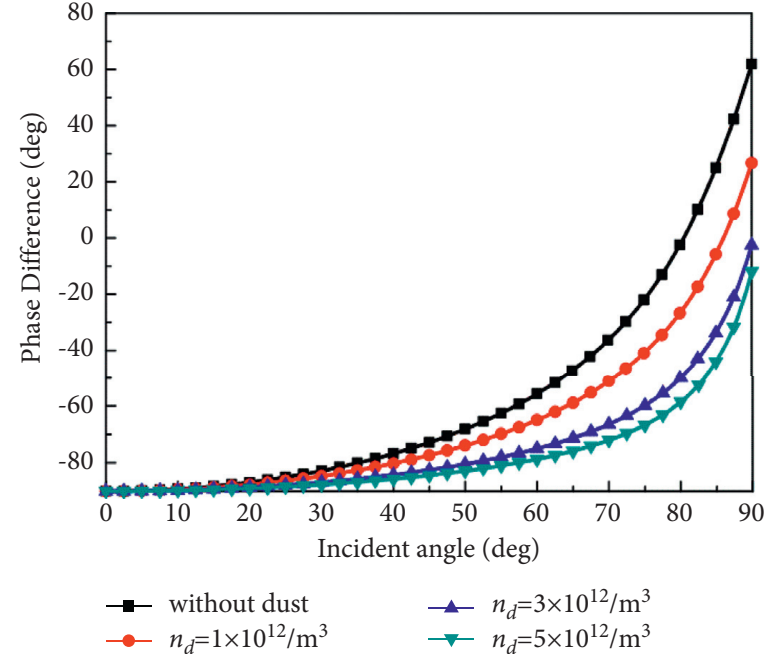

(b)

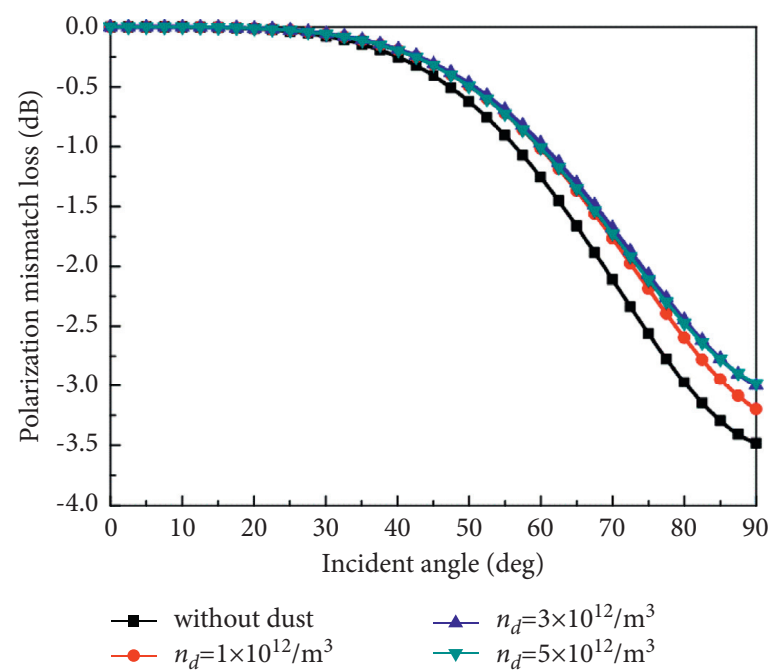

(c)

Figure 4: Effects of different dust densities on EM waves versus the incident angle: (a) axial ratio, (b) phase difference, and (c) polarization mismatch loss.

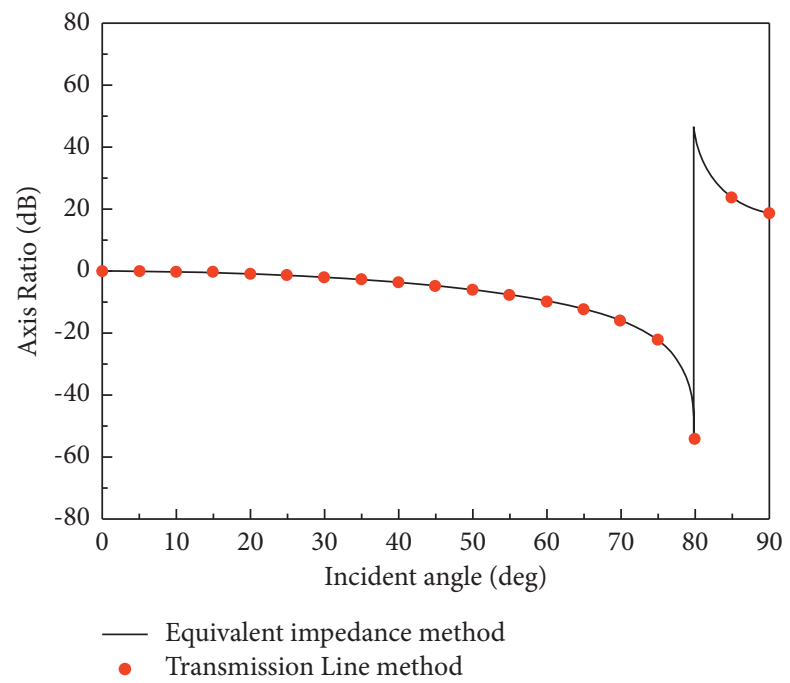

Figure 5: Comparison of calculation results for axial ratio. 


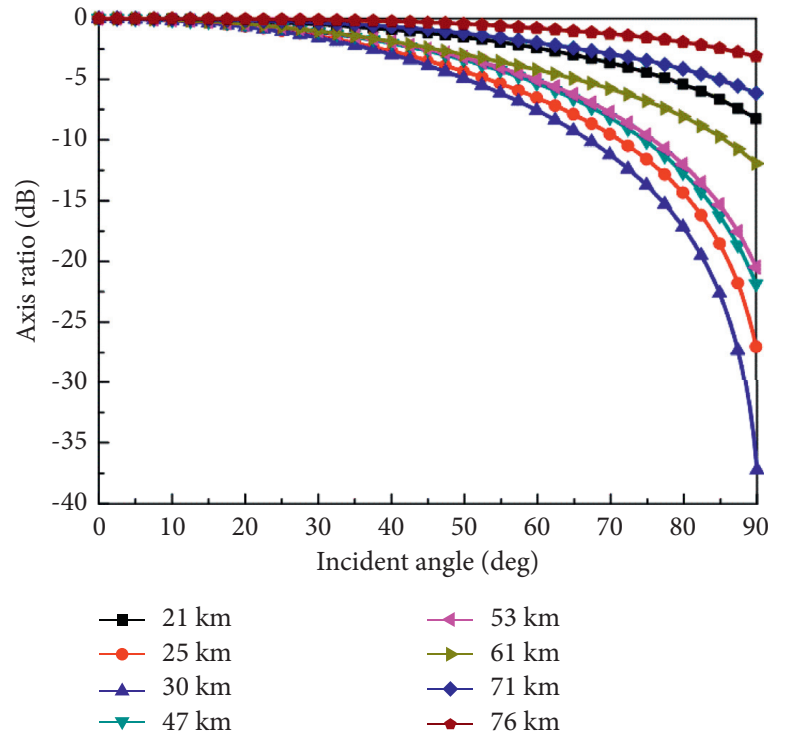

(a)

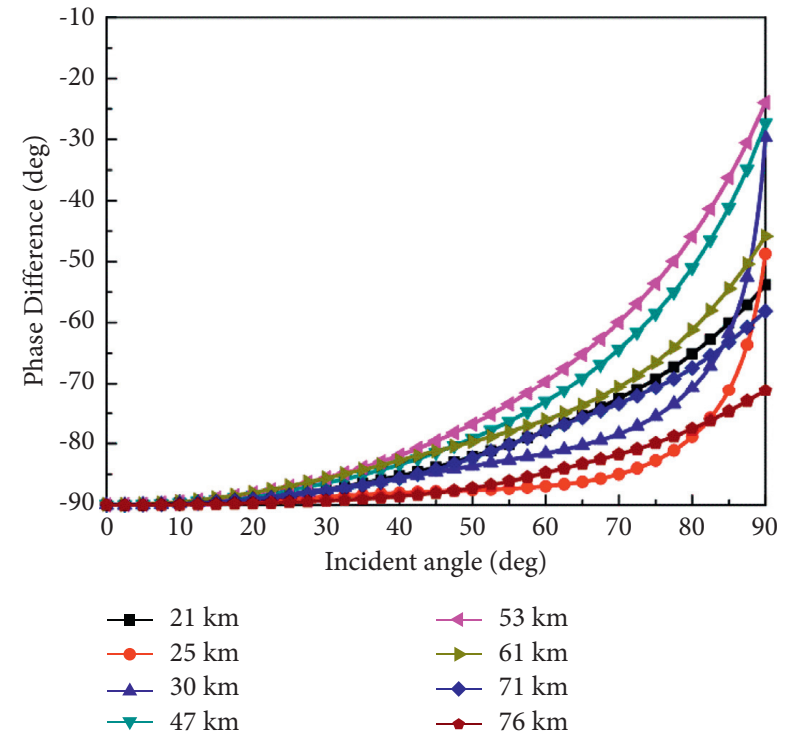

(b)

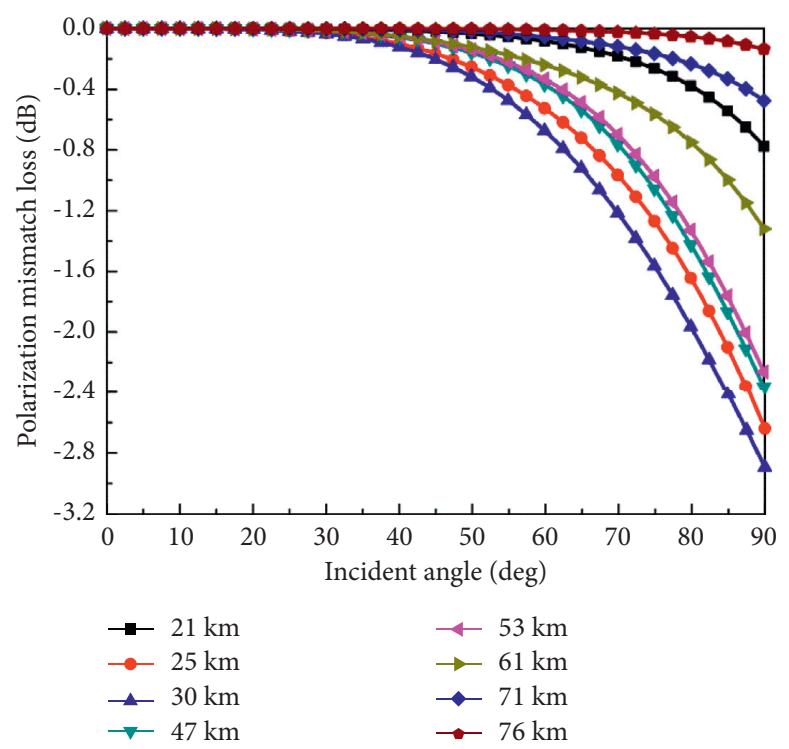

(c)

Figure 6: Effects of different reentry heights on EM waves versus the incident angle: (a) axial ratio, (b) phase difference, and (c) polarization mismatch loss.

combination with the electron density in the NASA experiment [29], this can be attributed to the spatial distribution of electron densities at each height. In addition, when the incident angle is less than $25^{\circ}$, the polarization state of the EM wave is slightly changed at each height, and the transmitted signals basically match with the polarization state of the receiving antenna.

\section{Conclusion}

This paper discussed the effects of inhomogeneous weakly ionized dusty plasma on the polarization properties of EM waves. The axial ratio, the phase difference, and the mismatch loss were calculated under different external magnetic fields and dust parameters. We further calculated the mismatch loss caused by inhomogeneous weakly ionized dusty plasma with different electron density distributions at different reentry heights. Based on these numerical results, some useful conclusions are made.

First, the external magnetic field aggravated the deterioration of polarization properties and caused more polarization mismatch loss. The larger the magnetized field is, the more transmitted waves cannot be received because of polarization mismatch. Second, the dust particles can alleviate the depolarization effect of EM waves and reduce the polarization mismatch loss. The phenomenon of polarization reversal can be avoided by regulating and controlling 
the radii and density of dust particles. Finally, the polarization state of the transmitted wave changes at eight reentry heights, namely, each height produces a certain polarization mismatch loss. The loss is the maximum at $30 \mathrm{~km}$ and the minimum at $76 \mathrm{~km}$. The problem of communication blackout may be caused by the polarization mismatch loss. These results can provide a theoretical reference for information reception in plasma containing ablative particles and other experimental research studies.

\section{Appendix}

For the perpendicular wave, the expressions of electric field and magnetic field in free space 1 (in Figure 1) are

$$
\begin{aligned}
& E_{0}=E_{\perp}^{1 i} e^{-j k_{1 i}\left(x \sin \theta_{1 i}+z \cos \theta_{1 i}\right)}+E_{\perp}^{1 r} e^{-j k_{1 r}\left(x \sin \theta_{1 r}-z \cos \theta_{1 r}\right)}, \\
& H_{0}=\frac{1}{Z_{0}^{\perp}}\left(E_{\perp}^{1 i} e^{-j k_{1 i}\left(x \sin \theta_{1 i}+z \cos \theta_{1 i}\right)}-E_{\perp}^{1 r} e^{-j k_{1 r}\left(x \sin \theta_{1 r}-z \cos \theta_{1 r}\right)}\right),
\end{aligned}
$$

where $Z_{0}^{\perp}=\sqrt{\mu_{0} / \varepsilon_{0}} \cdot \sec \theta_{1 i}$ is the wave impedance of the perpendicular polarized wave in free space 1 . The expressions of electric field and magnetic field in stratified plasma are

$$
\begin{aligned}
& E_{m t}=E_{\perp}^{m t} e^{-j k_{m t}\left(z-\sum_{i=1}^{n} d_{i}\right) \cos \theta_{m t}} e^{-k_{m t} x \sin \theta_{m t}}+E_{\perp}^{m r} e^{j k_{m r}\left(z-\sum_{i=1}^{n} d_{i}\right) \cos \theta_{m t}} e^{-k_{m t} x \sin \theta_{m t}}, \\
& H_{m t}=\frac{1}{Z_{m}^{\perp}}\left(E_{\perp}^{m t} e^{-j k_{m t}\left(z-\sum_{i=1}^{n} d_{i}\right) \cos \theta_{m t}} e^{-k_{m t} x \sin \theta_{m t}}-E_{\perp}^{m r} e^{j k_{m r}\left(z-\sum_{i=1}^{n} d_{i}\right) \cos \theta_{m t}} e^{-k_{m t} x \sin \theta_{m t}}\right),
\end{aligned}
$$

where $Z_{m}^{\perp}=\sqrt{\mu_{m} / \varepsilon_{m}} \cdot \sec \theta_{m i}$ is the wave impedance of the perpendicular polarized wave at layer $m, m=2,3, \ldots n$. $\theta_{m t}$ is the transmitted angle in this layer and can be obtained by Snell's Law.

The electric field and magnetic field of the perpendicular polarized wave in free space 2 are

$$
\begin{aligned}
& E_{(n+1) t}=E_{\perp}^{(n+1) t} e^{-j k_{(n+1) t}\left(z-\sum_{i=1}^{n} d_{i}\right) \cos \theta_{n t}} e^{-k_{(n+1) t} x \sin \theta_{n t}}, \\
& H_{(n+1) t}=\frac{1}{Z_{n+1}} \cdot E_{\perp}^{(n+1) t} e^{-j k_{(n+1) t}\left(z-\sum_{i=1}^{n} d_{i}\right) \cos \theta_{n t}} e^{-k_{(n+1) t} x \sin \theta_{n t}} .
\end{aligned}
$$

According to the boundary condition, the electric field and magnetic field are continuous. So, $E_{m i}=E_{(m+1) i}$ and $H_{m i}=H_{(m+1) i}$ in the places where $z=d_{i}$. Thus, the reflected coefficients and transmitted coefficients of the perpendicular polarized wave at layer $m$ can be obtained:

$$
\begin{aligned}
& R_{\perp}^{m}=\frac{Z_{e f m}^{\perp} \cos \theta_{m i}-Z_{m}^{\perp} \cos \theta_{(m+1) i}}{Z_{e f m}^{\perp} \cos \theta_{m i}+Z_{m}^{\perp} \cos \theta_{(m+1) i}}, \\
& T_{\perp}^{m}=\frac{1+R_{\perp}^{m}}{1+R_{\perp}^{m} e^{-j 2 k_{m+1} d_{m+1} \cos \theta_{(m+1) i}}},
\end{aligned}
$$

where $Z_{\text {efm }}^{\perp}$ is the equivalent impedance of the perpendicular polarized wave at layer $m$.

$$
\begin{aligned}
Z_{e f m}^{\perp} & =Z_{m}^{\perp} \frac{Z_{e f(m+1)}^{\perp} \cos \theta_{m i}+j Z_{m}^{\perp} \cos \theta_{(m+1) i} \tan \left(k_{m} d_{m} \cos \theta_{m i}\right)}{Z_{m}^{\perp} \cos \theta_{(m+1) i}+j Z_{e f(m+1)}^{\perp} \cos \theta_{m i} \tan \left(k_{m} d_{m} \cos \theta_{m i}\right)}, \\
Z_{e f(n+1)}^{\perp} & =Z_{1}^{\perp} .
\end{aligned}
$$

In the same way, the reflected coefficients and transmitted coefficients of the parallel polarized wave at layer $m$ are

$$
\begin{aligned}
R^{m} & =\frac{Z_{m} \cos \theta_{m i}-Z_{e f(m+1)} \cos \theta_{(m+1) i}}{Z_{m} \cos \theta_{m i}+Z_{e f(m+1)} \cos \theta_{(m+1) i}}, \\
T^{m} & =\frac{1-R^{m}}{1-R^{m+1} e^{-j 2 k_{m+1} d_{m+1} \cos \theta_{(m+1) i}}} \cdot \frac{\cos \theta_{m i}}{\cos \theta_{(m+1) i}},
\end{aligned}
$$

where $Z_{e f m}$ is the equivalent impedance of the parallel polarized wave at layer $m$.

$$
Z_{e f m}=Z_{m} \frac{Z_{e f(m+1)} \cos \theta_{(m+1) i}+j Z_{m} \cos \theta_{m i} \tan \left(k_{m} d_{m} \cos \theta_{m i}\right)}{Z_{m} \cos \theta_{(m+1) i}+j Z_{e f(m+1)} \cos \theta_{(m+1) i} \tan \left(k_{m} d_{m} \cos \theta_{m i}\right)} .
$$

In (A.7), $Z_{e f(n+1)}^{\|}=Z_{1}^{\|}$, and $Z_{m}^{\|}=\sqrt{\mu_{m} / \varepsilon_{m}} \cdot \cos \theta_{m i}$ is the wave impedance of the parallel polarized wave at layer $m$.

\section{Data Availability}

The data that supported the findings of this study are available from the corresponding author upon reasonable request.

\section{Conflicts of Interest}

The authors declare that they have no conflicts of interest.

\section{Acknowledgments}

This work was supported by the National Natural Science Foundation of China (Grant nos. U20B2059, 62071353, 61627901, and 62071348), the Foundation for Innovative Research Groups of the National Natural Science Foundation of China (Grant no. 61621005), the Key Laboratory 
Foundation (Grant no. 6142502190203), and the Chinese Postdoctoral Science Foundation (Grant no. 2019M653544).

\section{References}

[1] K. Kinefuchi, I. Funaki, T. Shimada, and T. Abi, "Computational fluid dynamics and frequency-dependent finite-difference time-domain method coupling for the interaction between microwaves and plasma in rocket plumes," Physics of Plasmas, vol. 19, no. 10, pp. 677-686, 2012.

[2] J. L. Poirier, W. Rotman, D. T. Hayes, and J. F. Lennon, "Effects of the reentry plasma sheath on microwave antenna performance: trailblazer II rocket results of 18 June 1967," Scientific Reports, Bedford, MA, USA, 1971.

[3] S. Dap, D. Lacroix, R. Hugon, and J. Bougdira, "Retrieving particle size and density from extinction measurement in dusty plasma, Monte Carlo inversion and Ray-tracing comparison," Journal of Quantitative Spectroscopy and Radiative Transfer, vol. 128, pp. 18-26, 2013.

[4] V. Y. Karasev, E. S. Dzlieva, S. I. Pavlov, L. A. Novikov, and I. C. Mashek, "Dusty plasma in a strongly inhomogeneous magnetic field," Technical Physics Letters, vol. 46, no. 4, pp. 371-373, 2020.

[5] Y. Liu, W. Chen, L. Yang, H. Zhixing, G. Linjing, and D. Qingqing, "Research on the propagation properties of $\mathrm{THz}$ circularly polarized wave in BGK model inhomogeneous dusty plasma," Physics of Plasmas, vol. 27, Article ID 093702, 2020.

[6] J. S. Jia, C. X. Yuan, S. Liu et al., "Propagation of electromagnetic waves in a weak collisional and fully ionized dusty plasma," Physics of Plasmas, vol. 23, Article ID 043302, 2016.

[7] W. Chen, L. X. Guo, J. T. Li, and S. H. Liu, "Research on the FDTD method of electromagnetic wave scattering characteristics in time-varying and spatially nonuniform plasma sheath," IEEE Transactions on Plasma Science, vol. 44, no. 22, pp. 3235-3242, 2016.

[8] W. Chen, L. Yang, and L. X. Guo, "Research on the propagation characteristics of $\mathrm{THz}$ waves in spatial inhomogeneous and time-varying and weakly ionized dusty plasma," IEEE Transactions on Plasma Science, vol. 47, no. 10, pp. 4745-4752, 2019.

[9] Y. Zhang, G. Xu, and Z. Zheng, "Propagation of terahertz waves in a magnetized, collisional, and inhomogeneous plasma with the scattering matrix method," Optik, vol. 182, pp. 618-624, 2019.

[10] J. Zhang, H. Fu, and W. Scales, "FDTD analysis of propagation and absorption in nonuniform anisotropic magnetized plasma slab," IEEE Transactions on Plasma Science, vol. 46, no. 6, pp. 2146-2153, 2018.

[11] B. Jazi, Z. Rahmani, and B. Shokri, "Reflection and absorption of electromagnetic wave propagation in an inhomogeneous dissipative magnetized plasma slab," IEEE Transactions on Plasma Science, vol. 41, no. 2, pp. 290-295, 2013.

[12] X. Yin, H. Zhang, S.-J. Sun, Z. Zhao, and Y.-L. Hu, "Analysis of propagation and polarization characteristics of electromagnetic waves through nonuniform magnetized plasma slab using propagator matrix method," Progress In Electromagnetics Research, vol. 137, pp. 159-186, 2013.

[13] A. Shahrokhi, H. Mehdian, K. Hajisharifi, and A. Hasanbeigi, "The polarization evolution of electromagnetic waves as a diagnostic method for a motional plasma," Applied Physics B, vol. 123, no. 12, p. 292, 2017.

[14] J. Shi, Y. Gao, J. Wang, Z. Yuan, and Y. Ling, "Electromagnetic reflection of conductive plane covered with magnetized inhomogeneous plasma," International Journal of Infrared and Millimeter Waves, vol. 22, no. 8, pp. 1167-1175, 2001.

[15] H. Li, J. Wu, Z. Zhou, and C. Yuan, "Propagation of electromagnetic wave in dusty plasma and the influence of dust size distribution," Physics of Plasmas, vol. 23, no. 7, Article ID 073702, 2016.

[16] P. K. Shukla and A. A. Mamun, Introduction to Dusty Plasma Physics, CRC Press, Boca Raton, FL, USA, 2001.

[17] J.-Z. Duan, J.-F. Han, C.-L. Wang et al., "Contribution of the dust grains to the damping of the electromagnetic waves propagating in plasma," IEEE Transactions on Plasma Science, vol. 41, no. 8, pp. 2434-2437, 2013.

[18] L. Lu and Z. Li, "Effect of dust charge fluctuations on kelvinhelmholtz instability in a cold dust plasma," Physics of Plasmas, vol. 7, p. 424, 2000.

[19] J. H. Joo Hwa Lee and D. K. Kalluri, "Three-dimensional FDTD simulation of electromagnetic wave transformation in a dynamic inhomogeneous magnetized plasma," IEEE Transactions on Antennas and Propagation, vol. 47, no. 7, pp. 1146-1151, 1999.

[20] J.-Z. Duan, C.-L. Wang, J.-R. Zhang et al., "Influence of charging process and size distribution of dust grain on the electric conductivity of dusty plasma," Physics of Plasmas, vol. 19, no. 8, Article ID 083703, 2012.

[21] D. B. Ge, Y. X. Shi, and J. Wu, "The research on the dielectric tensor of weakly ionized dust plasma," Acta Physica Sinica, vol. 58, no. 8, pp. 5507-5512, 2019.

[22] R. Rawhouser, "Overview of the AF avionics laboratory reentry EMs program," Tech. Rep. NASA/SP-252, NASA, Washington, DC, USA, 1971.

[23] G. Cerri, F. Moglie, R. Montesi, P. Russo, and E. Vecchioni, "FDTD solution of the maxwell-Boltzmann system for electromagnetic wave propagation in a plasma," IEEE Transactions on Antennas and Propagation, vol. 56, no. 8, pp. 2584-2588, 2008.

[24] N. D'Angelo, "Electrostatic dust-cyclotron waves in plasmas with opposite polarity grains," Planetary and Space Science, vol. 50, no. 4, pp. 375-378, 2002.

[25] D. Liu, "Study on the low-Frequency EM wave transmission characteristics under plasma sheath and blackout mitigation by $\mathrm{E} \times \mathrm{B}$," M.S. dissertation, Xidian University., Xi'an, China, 2015.

[26] X. Wang, "Study on polarization information processing," M.S. dissertation, National University of Defense Technology., Hunan, China, 1999.

[27] D. M. Dix, "Typical values of plasma parameters around a conical re-entry vehicle," Scientific Reports AD295429, Aerospace Corporation, EI Segundo, CA, USA, 1962.

[28] B. Bai, X. Li, Y. Liu, J. Xu, L. Shi, and K. Xie, "Effects of reentry plasma sheath on the polarization properties of obliquely incident EM waves," IEEE Transactions on Plasma Science, vol. 42, no. 10, pp. 3365-3372, 2014.

[29] C. T. Swift, J. T. Beck, J. Thomson, and S. L. Castellow, RAM C-III S-BAND Diagnostic Experiment, NASA, Washington, DC, USA, 1970. 